
\title{
R Reserach S Suare \\ Serum Metabolomic Patterns in Young Patients with Ischemic Stroke: A Case Control Study
}

Jia Liu

Beijing Chaoyang Hospital

Junliang Yuan

Beijing Chaoyang Hospital

Jingwei Zhao

Beijing Chaoyang Hospital

Lin Zhang

Beijing Chaoyang Hospital

Qiu Wang

Beijing Chaoyang Hospital

Guang Wang ( $\nabla$ drwg6688@126.com )

Beijing Chaoyang Hospital https://orcid.org/0000-0002-2328-569X

Research

Keywords: serum metabolomics, ischemic stroke, young adult

Posted Date: August 12th, 2020

DOI: https://doi.org/10.21203/rs.3.rs-55452/v1

License: (c) (i) This work is licensed under a Creative Commons Attribution 4.0 International License.

Read Full License 


\section{Abstract}

Background: Ischemic stroke is one of the leading causes of death and adult disability. The incidence of ischemic stroke continues to rise in young adults. This study aimed to provide a comprehensive evaluation of metabolic changes and explore possible mechanisms in young ischemic stroke patients without common risk factors.

Methods: This study investigated serum metabolomics in 50 young patients with newly suffered ischemic stroke and 50 age-, sex-, and body mass index-matched healthy controls. The metabolomic data were analyzed by performing a multivariate statistical analysis.

Results: The 197 metabolites, including amino acids, bile acids, free fatty acids, and lipids, were identified in all participants. Multivariate models showed significant differences in serum metabolomic patterns between young patients with ischemic stroke and healthy controls. The stroke patients had increased Lmethionine, homocysteine, glutamine, uric acid, GCDCA, and PE (18:0/20:4, 16:0/22:5), and decreased levels of L-citrulline, taurine, PC (16:2/22:6, 16:2/20:5, 15:0/18:2), and SM (d18:1/23:0, d20:0/19:1, d18:1/22:0, d16:0/26:1, d16:0/18:0, d16:0/22:1, d18:1/19:1, d16:0/17:1, d16:1/24:1, d18:1/19:0). Based on the identified metabolites, the metabolic pathways of arginine biosynthesis, glycerophospholipid metabolism, and taurine and hypotaurine metabolism were significantly enriched in the young patients with ischemic stroke.

Conclusions: Serum metabolomic patterns were significantly different between young patients with ischemic stroke and healthy controls.

\section{Background}

Ischemic stroke is one of the leading causes of death and adult disability [1]. In recent years, the incidence of ischemic stroke has declined gradually in the general population but has continued to rise in young adults [2]. Most patients cannot completely recover even after proper treatment, so ischemic stroke has major social and economic impacts for working-age adults. Management of stroke risk factors is considered as the best strategy to decrease the incidence of ischemic stroke [2]. Notably, the etiology of ischemic stroke differs significantly between young and older patients [2-4]. Several previous studies have indicated that smoking, hypertension, and diabetes are common risk factors in young patients with ischemic stroke [5-6]. Some cardiac and vascular diseases, such as arrhythmia, cerebral artery dissection, and small vessel disease, were also common causes in young ischemic stroke patients [2-4]. Although several risk factors have been discovered, over a third of ischemic strokes in young adults remain cryptogenic, which hints at an inadequate understanding of the pathogenesis of ischemic stroke in young adults [2-4]. Therefore, identifying novel risk factors and understanding the pathogenesis of ischemic stroke in young adults is urgent and important.

Metabolomics is a novel analytical approach dedicated to specifically identifying small-molecule metabolites, which represents the endpoint of the omics cascade and provides an explanation of the 
pathophysiology and metabolic changes of some diseases. Several studies have identified altered metabolomics in patients with ischemic stroke; however, few studies have focused on young patients [710]. This study aims to provide a comprehensive evaluation of metabolic changes and explore possible mechanisms in young ischemic stroke patients without common risk factors.

\section{Methods}

\section{Study design and participants}

A total of 50 patients between ages 18 and 50 who newly suffered ischemic stroke were consecutively recruited between September 2016 and October 2017 at the Beijing Chaoyang Hospital Affiliated with Capital Medical University [11]. Meanwhile, 50 age-, sex-, and body weight index (BMI)-matched healthy controls were enrolled from the Physical Examination Center at the same hospital. Ischemic stroke was diagnosed by clinical symptoms and imaging examination, including a cranial computed tomography scan and/or magnetic resonance imaging within 24 hours of hospital admission, according to the International Classification of Diseases, 10 th revision. All healthy controls had a normal cranial imaging examination and no history of cerebrovascular diseases according to the medical history collection and physical examination. All participants in the control and stroke groups had no history of diabetes, hypertension, hypercholesterolemia, past or present cigarette smoking, pregnancy and puerperium, use of vasoactive or conceptive or illicit drugs, excessive drinking, heart or vascular disease, atrial fibrillation or flutter, cervical arterial dissection, hematologic disease, antiphospholipid antibody syndrome, infectious disease, cardiac or liver or renal function impairment, thyroid dysfunction, systemic inflammatory disease, or cancer. All participants in the control and stroke groups had no family history of coronary heart disease or stroke, which was defined as coronary artery disease, sudden death, or stroke in a firstdegree male relative younger than 55 years old or a female relative younger than 65 years old. All studies were conducted in accordance to the principles of the Declaration of Helsinki. The study protocol was approved by the Ethics Committee of Beijing Chaoyang Hospital Affiliated with Capital Medical University (application number: 2016-K103). Each participant provided written informed consent to be included in the study.

\section{Clinical tests}

A standard questionnaire completed by two skilled nurses was used to collect information on the demographics, lifestyle characteristics, health status, and medications of each participant. Height and weight were measured by the same trained group to the nearest $0.1 \mathrm{~cm}$ and $0.1 \mathrm{~kg}$, respectively. Blood samples were obtained after an overnight fasting within 7 days of the onset of ischemic stroke symptoms. Clinical parameters were measured immediately. Serums were stored at $-80^{\circ} \mathrm{C}$ for metabolomic analysis. Parameters, including total cholesterol (TC), high-density lipoprotein cholesterol (HDL-C), low-density lipoprotein cholesterol (LDL-C), and triglycerides (TG), were measured enzymatically as described previously (12). The levels of total bile acid (TBA) were measured using an enzymatic cycling method (Hitachi 747, Roche Diagnostics, Germany). Uric acid (UA) was measured using the 
enzymatic uricase method (Hitachi 747, Roche Diagnostics, Germany). HbA1c was measured using the HPLC method. Homocysteine was measured using the cycling enzymatic method (Hitachi 747, Roche Diagnostics, Germany). BMI was calculated as weight in kilograms divided by height in meters squared. All stroke patients were examined to exclude common risk factors for ischemic stroke via electrocardiograph, transesophageal and transthoracic echocardiogram, brain magnetic resonance angiography, carotid and vertebral computed tomography angiography, immunological antibodies (such as antinuclear antibodies, lupus anticoagulants, anticardiolipin antibodies, etc.), and serum functional levels of antithrombin, protein $\mathrm{C}$, and protein $\mathrm{S}$, etc. Individuals were considered to have dyslipidemia if they had a total cholesterol level over $200 \mathrm{mg} / \mathrm{dL}$, or if they were being already treated. Individuals were considered to have diabetes if they had $\mathrm{HbA} 1 \mathrm{c}$ levels $\geq 6.5 \%$, or if they were already diagnosed as diabetic. Individuals were considered to have hypertension if they had ever had diastolic blood pressure $\geq 90 \mathrm{mmHg}$ and/or systolic blood pressure $\geq 140 \mathrm{mmHg}$ and/or used antihypertensive medication.

\section{Metabolomics}

The detailed method for metabolomic analyses has been described previously [12]. In brief, we used liquid chromatography of Waters I-Class (Waters, Milford, MA, USA) coupled with a Waters Xevo TQ-S (Waters, Milford, MA, USA) mass spectrometer with an electrospray ionization (ESI) source to analyze each amino acid or bile acid extract. Each free fatty acid or lipid extract was analyzed by liquid chromatography using an LC-20AXR Rapid Separation LC system (Shimadzu, Kyoto, Japan) coupled with a Qtrap5500 (AB SCIEX, Redwood City, CA, USA) mass spectrometer with an ESI source. MassLynx software 4.1 (Waters, USA) and Analysis software (SCIEX, USA) were used for systemic control and data acquisition, respectively. The samples from the control and stroke groups were alternately injected into the analytic workflow at a random order. The quality control samples, which were mixed with equal aliquots of serums from the control and stroke groups, were injected into every 15 samples throughout the analytical workflow. Skyline software (MacCoss, University of Washington) was used to analyze ultrahigh-performance liquid chromatography-mass spectrometry raw data and obtain the quantitative concentration of each metabolite in the samples. The features were selected based on their coefficients of variation (CVs) with quality control (QC) samples. The features with CVs over $15 \%$ were excluded. The stability and reliability of metabolomic data were evaluated prior to data analysis, and the results showed that the method was excellent (Supplementary Figures S1-S4).

\section{Statistical analysis}

The clinical parameters of the control and stroke groups are expressed by means \pm standard deviations for normally distributed data. TBA variables had a skewed distribution, and are shown as median and upper and lower quartiles. The skewed-distribution variables were log-transformed before analysis. The differences between the control and stroke groups were analyzed using an independent sample t-test or a Mann-Whitney $U$ test. The proportions were analyzed using chi-squared tests. All statistical analyses were performed using SPSS 21.0 (Chicago, IL, USA). All tests were two-tailed, and the results were considered statistically significant when $P<0.05$. 
Metabolomic data were analyzed using SIMCA 14.0 (Umetrics AB, Umeå, Sweden) and MetaboAnalyst 4.0 (www.metaboanalyst.ca) [13]. MetaboAnalyst 4.0 was used to normalize data, reduce systematic bias, and improve consistency. The metabolites with features $>50 \%$ missing values were removed. The remaining missing values were replaced by the half of the minimum positive value in the original data. Both a principal component analysis (PCA) and an orthogonal partial least-squares discriminant analysis (OPLS-DA) were performed to reveal the global metabolic changes between the control and stroke groups, using SIMCA 14.0. A validation plot was used to assess the validity of the OPLS-DA model using sevenfold cross-validation and permutation tests $(n=200)$. The variable influences on projection $(V I P)$ values were calculated using the OPLS-DA model. The serum differential variables with VIP values $>1.5$ from the OPLS-DA model were assessed using Student's $t$-test or the Wilcoxon (Mann-Whitney U) test to analyze significance. Significant differences in the pathway were evaluated using the hypergeometric test, and pathway topology was analyzed based on the relative betweenness centrality.

\section{Results}

\section{Baseline characteristics of the control and stroke groups}

Table 1 presents the baseline characteristics of the control and stroke groups. The two groups were well matched for age, gender, and BMI. The stroke group had higher TG, UA, and homocysteine and lower HDL-C levels (all $P<0.01$ ). There was no significant difference in TC, LDL-C, TBA, or HbA1c between the control and stroke groups. 
Table 1

Baseline characteristics of the control and stroke groups

\begin{tabular}{|c|c|c|c|}
\hline Parameters & $\begin{array}{l}\text { Control } \\
(n=50)\end{array}$ & $\begin{array}{l}\text { Stroke } \\
(n=50)\end{array}$ & $P$ \\
\hline Age,y & $42.8 \pm 4.3$ & $43.4 \pm 6.3$ & .609 \\
\hline Gender, F/M, n & $38 / 12$ & $38 / 12$ & \\
\hline $\mathrm{BMI}, \mathrm{kg} / \mathrm{m}^{2}$ & $22.76 \pm 3.66$ & $23.54 \pm 1.88$ & .411 \\
\hline $\mathrm{TC}, \mathrm{mmol} / \mathrm{L}$ & $4.72 \pm 0.58$ & $4.49 \pm 1.01$ & .185 \\
\hline $\mathrm{HDL}-\mathrm{C}, \mathrm{mmol} / \mathrm{L}$ & $1.45 \pm 0.38$ & $1.10 \pm 0.29$ & .000 \\
\hline LDL-C, mmol/L & $2.68 \pm 0.40$ & $2.48 \pm 0.68$ & .103 \\
\hline $\mathrm{TG}, \mathrm{mmol} / \mathrm{L}$ & $1.14 \pm 0.43$ & $2.05 \pm 1.39$ & .000 \\
\hline TBA, $\mu \mathrm{mol} / \mathrm{L}$ & $2.60(1.80-4.10)$ & $3.00(1.70-4.90)$ & .465 \\
\hline $\mathrm{UA}, \mu \mathrm{mol} / \mathrm{L}$ & $280.65 \pm 62.54$ & $338.43 \pm 94.34$ & .001 \\
\hline $\mathrm{HbA} 1 \mathrm{c}, \%$ & $5.55 \pm 0.51$ & $5.41 \pm 0.41$ & .241 \\
\hline Homocysteine, $\mu \mathrm{mol} / \mathrm{L}$ & $15.95 \pm 1.75$ & $17.09 \pm 5.99$ & .037 \\
\hline
\end{tabular}

\section{Metabolomic analysis of the control and stroke groups}

The 197 metabolites were identified in all participants (Table S1). PCA score plots showed a clear clustering of the control and stroke groups, and the cumulative fitness (R2 value) of the PCA model was 0.767 (Figure. 1). The OPLS-DA analysis indicated clear separations between the control (green dots) and stroke (blue dots) groups $(\mathrm{R} 2 \mathrm{Y}=0.928, \mathrm{Q} 2=0.814$, Fig. $2 \mathrm{~A})$. The results of the permutation test strongly indicate that the original model was valid ( $R 2$ intercept $=0.252$, Q2 intercept $=-0.394$, Fig. $2 B$ ).

Based on the selection criteria (VIP > 1.5 and $P<0.05)$, 20 metabolites were obtained (Table 2). Compared to the healthy controls, the stroke group had higher levels of L-methionine, glutamine, glycochenodeoxycholic acid (GCDCA), and phosphatidylethanolamine (PE) (18:0/20:4, 16:0/22:5) (Fig. 3A) (Table 2). Decreased levels of L-citrulline, taurine, phosphatidylcholine (PC) (16:2/22:6, 16:2/20:5, 15:0/18:2), and sphingomyelin (SM) (d18:1/23:0, d20:0/19:1, d18:1/22:0, d16:0/26:1, d16:0/18:0, d16:0/22:1, d18:1/19:1, d16:0/17:1, d16:1/24:1, d18:1/19:0) were observed in the stroke group compared to the control group (Fig. 3B) (Table 2). 
Table 2

Significant changed metabolites of the stroke patients

\begin{tabular}{|c|c|c|}
\hline Metabolites & VIP & $P$ \\
\hline L-Citrulline & 4.12 & .000 \\
\hline $\mathrm{PC}(16: 2 / 22: 6)$ & 3.49 & .000 \\
\hline PC (16:2/20:5) & 2.42 & .000 \\
\hline SM (d18:1/23:0) & 2.05 & .000 \\
\hline SM (d20:0/19:1) & 1.98 & .000 \\
\hline SM (d18:1/22:0) & 1.95 & .000 \\
\hline SM (d16:0/26:1) & 1.90 & .000 \\
\hline L-Methionine & 1.85 & .000 \\
\hline SM (d16:0/18:0) & 1.81 & .000 \\
\hline SM (d16:0/22:1) & 1.77 & .000 \\
\hline PE (18:0/20:4) & 1.66 & .000 \\
\hline SM (d18:1/19:1) & 1.64 & .002 \\
\hline PE (16:0/22:5) & 1.64 & .000 \\
\hline Glutamine & 1.64 & .000 \\
\hline SM (d16:0/17:1) & 1.63 & .000 \\
\hline SM (d16:1/24:1) & 1.59 & .009 \\
\hline GCDCA & 1.55 & .012 \\
\hline Taurine & 1.54 & .000 \\
\hline SM (d18:1/19:0) & 1.53 & .002 \\
\hline PC (15:0/18:2) & 1.52 & .000 \\
\hline
\end{tabular}

\section{Pathway analysis}

We further performed a pathway analysis to identify the significantly changed metabolic pathway, according to the Kyoto Encyclopedia of Genes and Genomes (KEGG) database. Based on the identified metabolites, the metabolic pathways of arginine biosynthesis, glycerophospholipid metabolism, and 
taurine and hypotaurine metabolism were significantly enriched in young patients with ischemic stroke (Fig. 4 and Table 3).

Table 3

The list of metabolic pathways with significant difference in the stroke group compared to the control group.

\begin{tabular}{|lllll|}
\hline Pathway name & Hits & $-\log (P)$ & FDR & Impact \\
\hline Arginine biosynthesis & 2 & 6.4687 & 0.1303 & 0.22843 \\
\hline Glycerophospholipid metabolism & 2 & 4.5815 & 0.3757 & 0.19895 \\
\hline Taurine and hypotaurine metabolism & 1 & 3.3342 & 0.42771 & 0.42857 \\
\hline Primary bile acid biosynthesis & 2 & 4.1068 & 0.3757 & 0.01735 \\
\hline Alanine, aspartate and glutamate metabolism & 1 & 2.1201 & 0.91653 & 0.11378 \\
\hline $\begin{array}{l}\text { Hit means the matched number in pathway; the } P \text { value is calculated from the enrichment analysis; } \\
\text { Impact value is calculated from pathway topography analysis; FDR value is the false discovery rate } \\
\text { adjusted } P \text { value. }\end{array}$ & & & \\
\hline
\end{tabular}

\section{Discussion}

The altered metabolomics in ischemic stroke have been identified in older patients by several studies; however, until now only a few studies focused on young patients (7-10). The present study showed that serum metabolomic patterns were significantly different between young patients with ischemic stroke and healthy controls. The young ischemic stroke patients had increased L-methionine, homocysteine, glutamine, uric acid, GCDCA and PE (18:0/20:4, 16:0/22:5) levels, and decreased levels of L-citrulline, taurine, PC (16:2/22:6, 16:2/20:5, 15:0/18:2), and SM (d18:1/23:0, d20:0/19:1, d18:1/22:0, d16:0/26:1, d16:0/18:0, d16:0/22:1, d18:1/19:1, d16:0/17:1, d16:1/24:1, d18:1/19:0). Based on the identified metabolites, the metabolic pathways of arginine biosynthesis, glycerophospholipid metabolism, and taurine and hypotaurine metabolism were significantly enriched in the young patients with ischemic stroke.

Amino acids are an important group of metabolites that participate in multiple physiological and pathophysiological processes. Consistent with previous studies in older people, the present study showed that young patients with ischemic stroke had significantly increased L-methionine, homocysteine, uric acid, and glutamine levels [14-17]. As is known, both hyperhomocysteinemia and hyperuricemia are independent risk factors for stroke [14-15]. As an essential amino acid, methionine comes from dietary intake. Homocysteine is an intermediate in methionine metabolism, and a moderate methionine diet of four weeks can induce hyperhomocysteinemia [18]. The present study found that young patients with ischemic stroke had significantly increased L-methionine. The elevated methionine and homocysteine levels might be associated with increased methionine intake. L-glutamine has been considered a beneficial amino acid that has antioxidant and anti-inflammatory effects [19-20]. L-glutamine 
supplementation reduced infarct volume and promoted neurobehavioral recovery in stroke mice [20]. And brain injury increased glutamine output in the glutamate-glutamine cycle, and further protected neurons from damage [21]. Therefore, increased L-glutamine might be a compensatory reaction to brain injury. Unlike results in older people, our study found that young patients with ischemic stroke had decreased levels of L-citrulline and taurine. Nitric oxide (NO) is a gas-signal molecule with various physiological functions, including regulating the balance of blood flow and oxygen demand and neurovascular coupling in the brain [22-23]. Endogenous NO was mainly generated from the citrulline-arginine-NO pathway [24]. Previous studies have shown that L-citrulline supplementation increased the bioavailability of L-arginine and promoted NO synthesis [24]. Taurine is a semiessential amino acid in mammals and has been proven to have multiple beneficial effects, including attenuating inflammation- and endoplasmic reticulum stress-induced organ injuries [25-26]. Taurine treatment inhibited ethanolmediated cell apoptosis in the cerebellum [27]. Therefore, decreased L-citrulline and taurine levels might be related to the pathogenesis of young patients with ischemic stroke.

As the key components of bile, bile acids are essential for regulating the digestion and absorption of dietary fat through the intestine. Recently, increasing evidence has shown that, beyond the gastrointestinal tract, circulating bile acids in the bloodstream also act as important signaling molecules for many pathophysiological processes [28-29]. The present study showed that the serum TBA levels were similar between the stroke and control groups, while the component of bile acid was significantly different. The present study showed that young patients with ischemic stroke had significantly increased GCDCA levels. GCDCA is a glycine-conjugated bile acid and has been demonstrated to be one of the most abundant bile acids in human serum [30-31]. GCDCA causes increased oxidative stress and promotes apoptosis by inducing JNK activation in rat hepatocytes [32]. Previous studies have shown that increased GCDCA levels are associated with liver injury induced by alcoholism or cholestasis [30-31]. Therefore, increased GCDCA levels might be related to the pathogenesis of young patients with ischemic stroke.

Several studies have demonstrated that lipid metabolites are associated with ischemic stroke [9, 33-34]. However, most previous studies were performed in older people, and their results also were controversial. The present study showed that lipid-related metabolites are significantly changed in young patients with ischemic stroke. PC, SM, and PE are all major constituents of cell membranes and play an important role in membrane-mediated cell signaling [35]. Previous studies have found that PC has many beneficial effects, including attenuating liver steatosis, slowing down aging-related processes, and improving brain function [36-37]. Consistent with previous research in older people, serum PCs were decreased in patients with ischemic stroke [33-34]. Moreover, the present study also found that young patients with ischemic stroke had increased PE (18:0/20:4, 16:0/22:5) levels and decreased levels of SM (d18:1/23:0, d20:0/19:1, d18:1/22:0, d16:0/26:1, d16:0/18:0, d16:0/22:1, d18:1/19:1, d16:0/17:1, d16:1/24:1, d18:1/19:0). SM and PE are abundant in brain, especially in the myelin sheet surrounding nerve cell axons [38-39]. Until now, the function of SM and PE remained unclear. Consistent with the present study, a recent study performed in three independent, follow-up, population-based cohorts also found a possible protective role for SM in stroke development [40]. The serum SM (32:1) level was demonstrated to 
inversely relate to the onset of ischemic stroke [40]. Further studies are needed to investigate whether changed levels of PC and PE are involved in the pathogenesis of young patients with ischemic stroke.

Besides the changed metabolites pattern, young patients with ischemic stroke had higher TG and FBG and lower HDL-C levels, when compared with age-, gender- and BMI-matched healthy controls. Consistent with the previous studies, relatively higher FBG within the normal range and increased TG and decreased HDL-C levels were observed in young patients with ischemic stroke but without a history of diabetes or hypercholesterolemia [9]. Therefore, more attention should be focused on young patients who have relatively higher FBG within the normal range and increased TG and decreased HDL-C levels.

The present study has some advantages and limitations. This was a case-control study, and the sample size was relatively small, which might limit the generalizability of the results. Our findings still warrant further studies to confirm our results. Ischemic stroke has an enormous influence on a working-age adult. Although several risk factors have been discovered, over a third of ischemic strokes in young adults remain cryptogenic. This study investigated metabolic differences and attempted to explore the possible mechanisms of ischemic stroke in young patients without common risk factors. Taken together, changes in metabolites might be involved in the pathogenesis of young patients with ischemic stroke. However, further independent validations, including human, animal, and cell experiments, are required before translating the results into clinical practice.

\section{Conclusions}

Serum metabolomic patterns were significantly different between young patients with ischemic stroke and healthy controls. Our study is beneficial in providing a further view into the pathophysiology of young patients with ischemic stroke. However, further independent validations, including human, animal, and cell experiments, are required to confirm these results and get better insight into the underlying mechanisms.

\section{Abbreviations}

$\mathrm{BMI}$

body weight index; TC:total cholesterol; HDL-C:high-density lipoprotein cholesterol; LDL-C:low-density lipoprotein cholesterol; TG:triglycerides; UA:uric acid; ESI:electrospray ionization; CV:coefficients of variation; QC:quality control; VIP:variable influences on projection; PCA:principal component analysis; OPLS-DA:orthogonal partial least-squares discriminant analysis; GCDCA:glycochenodeoxycholic acid; PE:phosphatidylethanolamine; PC:phosphatidylcholine; SM:sphingomyelin; KEGG:Kyoto Encyclopedia of Genes and Genomes; NO:nitric oxide

\section{Declarations}

\section{Ethics approval and consent to participate}


The study protocol was approved by the Ethics Committee of Beijing Chaoyang Hospital Affiliated with Capital Medical University (application number: 2016-K103). Each participant provided written informed consent to be included in the study.

\section{Consent for publication}

Not applicable

\section{Availability of data and materials}

The datasets used and/or analysed during the current study are available from the corresponding author on reasonable request.

\section{Competing interests}

The authors declare that they have no competing interests

\section{Funding:}

This work was supported by grants from the Beijing Talents foundation (2018-12) and Capital Funds for Health Improvement and Research (No. 2018-4-2034) to J.L.; and the Chinese National Natural Science Foundation (No. 81770792) and Key Projects of Science and Technology Planning of Beijing Municipal Education Commission (KZ201810025038) to G.W.

\section{Authors' contributions}

GW conceived and designed the experiments; JL, JLY, and JWZ performed the experiments; JL, JLY, LZ and QW analyzed and interpreted the patient data; JL and JLY wrote the manuscript; all authors read and approved the final manuscript.

\section{Acknowledgements}

Not applicable

\section{References}

1. Mortality GBD, Causes of Death C. Global, regional, and national life expectancy, all-cause mortality, and cause-specific mortality for 249 causes of death, 1980-2015: a systematic analysis for the Global Burden of Disease Study 2015. Lancet. 2016;388(10053):1459-544.

2. Ekker MS, Boot EM, Singhal AB, Tan KS, Debette S, Tuladhar AM, et al. Epidemiology, aetiology, and management of ischaemic stroke in young adults. Lancet Neurol. 2018;17(9):790-801.

3. Crespo Pimentel B, Willeit J, Toll T, Kiechl S, Pinho EMT, Canhao P, et al. Etiologic Evaluation of Ischemic Stroke in Young Adults: A Comparative Study between Two European Centers. J Stroke Cerebrovasc Dis. 2019;28(5):1261-6. 
4. McCarty JL, Leung LY, Peterson RB, Sitton CW, Sarraj A, Riascos RF, et al. Ischemic Infarction in Young Adults: A Review for Radiologists. Radiographics. 2019;39(6):1629-48.

5. Siriratnam P, Godfrey A, O'Connor E, Pearce D, Hu CC, Low A, et al. Prevalence and risk factors of ischemic stroke in the young; a regional Australian perspective. Intern Med J. 2019.

6. Maaijwee NA, Rutten-Jacobs LC, Schaapsmeerders P, van Dijk EJ, de Leeuw FE. Ischaemic stroke in young adults: risk factors and long-term consequences. Nat Rev Neurol. 2014;10(6):315-25.

7. Ke C, Pan CW, Zhang Y, Zhu X, Zhang Y. Metabolomics facilitates the discovery of metabolic biomarkers and pathways for ischemic stroke: a systematic review. Metabolomics. 2019;15(12):152.

8. Sun D, Tiedt S, Yu B, Jian X, Gottesman RF, Mosley TH, et al. A prospective study of serum metabolites and risk of ischemic stroke. Neurology. 2019;92(16):e1890-e8.

9. Holmes MV, Millwood IY, Kartsonaki C, Hill MR, Bennett DA, Boxall R, et al. Lipids, Lipoproteins, and Metabolites and Risk of Myocardial Infarction and Stroke. J Am Coll Cardiol. 2018;71(6):620-32.

10. Wang D, Kong J, Wu J, Wang X, Lai M. GC-MS-based metabolomics identifies an amino acid signature of acute ischemic stroke. Neurosci Lett. 2017;642:7-13.

11. Ekker MS, Verhoeven JI, Vaartjes I, van Nieuwenhuizen KM, Klijn CJM, de Leeuw FE. Stroke incidence in young adults according to age, subtype, sex, and time trends. Neurology. 2019;92(21):e2444-e54.

12. Liu J, Fu J, Jia Y, Yang N, Li J, Wang G. Serum Metabolomic Patterns in Patients with Autoimmune Thyroid Disease. Endocr Pract. 2020;26(1):82-96.

13. Chong J, Wishart DS, Xia J. Using MetaboAnalyst 4.0 for Comprehensive and Integrative Metabolomics Data Analysis. Curr Protoc Bioinformatics. 2019;68(1):e86.

14. Niazi F, Aslam A, Khattak S, Waheed S. Frequency of Homocysteinemia in Young Ischemic Stroke Patients and Its Relationship with the Early Outcome of a Stroke. Cureus. 2019;11(9):e5625.

15. Li J, Muraki I, Imano H, Cui R, Yamagishi K, Umesawa M, et al. Serum uric acid and risk of stroke and its types: the Circulatory Risk in Communities Study (CIRCS). Hypertens Res. 2020.

16. Liu M, Zhou K, Li H, Dong X, Tan G, Chai Y, et al. Potential of serum metabolites for diagnosing poststroke cognitive impairment. Mol Biosyst. 2015;11(12):3287-96.

17. Jove M, Mauri-Capdevila G, Suarez I, Cambray S, Sanahuja J, Quilez A, et al. Metabolomics predicts stroke recurrence after transient ischemic attack. Neurology. 2015;84(1):36-45.

18. Pexa A, Boeger RH, Henle T, Schwedhelm E, Deussen A. Effects of moderate hyperhomocysteinaemia induced by 4 weeks methionine-enriched diet on metabolite profile and mesenteric artery function in rats. Br J Nutr. 2008;99(5):993-9.

19. Zabot GP, Carvalhal GF, Marroni NP, Hartmann RM, da Silva VD, Fillmann HS. Glutamine prevents oxidative stress in a model of mesenteric ischemia and reperfusion. World J Gastroenterol. 2014;20(32):11406-14.

20. Luo LL, Li YF, Shan HM, Wang LP, Yuan F, Ma YY, et al. L-glutamine protects mouse brain from ischemic injury via up-regulating heat shock protein 70. CNS Neurosci Ther. 2019;25(9):1030-41. 
21. Ramonet D, Rodriguez MJ, Fredriksson K, Bernal F, Mahy N. In vivo neuroprotective adaptation of the glutamate/glutamine cycle to neuronal death. Hippocampus. 2004;14(5):586-94.

22. Garry PS, Ezra M, Rowland MJ, Westbrook J, Pattinson KT. The role of the nitric oxide pathway in brain injury and its treatment-from bench to bedside. Exp Neurol. 2015;263:235-43.

23. Liu Y, Croft KD, Hodgson JM, Mori T, Ward NC. Mechanisms of the protective effects of nitrate and nitrite in cardiovascular and metabolic diseases. Nitric Oxide. 2020;96:35-43.

24. Allerton TD, Proctor DN, Stephens JM, Dugas TR, Spielmann G, Irving BA. I-Citrulline Supplementation: Impact on Cardiometabolic Health. Nutrients. 2018;10(7).

25. Kim YS, Kim EK, Hwang JW, Kim JS, Shin WB, Dong X, et al. Neuroprotective Effect of Taurine-Rich Cuttlefish (Sepia officinalis) Extract Against Hydrogen Peroxide-Induced Oxidative Stress in SH-SY5Y Cells. Adv Exp Med Biol. 2017;975 Pt 1:243 - 54.

26. Jakaria M, Azam S, Haque ME, Jo SH, Uddin MS, Kim IS, et al. Taurine and its analogs in neurological disorders: Focus on therapeutic potential and molecular mechanisms. Redox Biol. 2019;24:101223.

27. Taranukhin AG, Taranukhina EY, Saransaari P, Podkletnova IM, Pelto-Huikko M, Oja SS. Neuroprotection by taurine in ethanol-induced apoptosis in the developing cerebellum. J Biomed Sci. 2010;17(Suppl 1):12.

28. Watanabe M, Houten SM, Mataki C, Christoffolete MA, Kim BW, Sato $\mathrm{H}$, et al. Bile acids induce energy expenditure by promoting intracellular thyroid hormone activation. Nature. 2006;439(7075):484-9.

29. Watanabe M, Houten SM, Wang L, Moschetta A, Mangelsdorf DJ, Heyman RA, et al. Bile acids lower triglyceride levels via a pathway involving FXR, SHP, and SREBP-1c. J Clin Invest. 2004;113(10):1408-18.

30. Luo L, Aubrecht J, Li D, Warner RL, Johnson KJ, Kenny J, et al. Assessment of serum bile acid profiles as biomarkers of liver injury and liver disease in humans. PLoS One. 2018;13(3):e0193824.

31. Yang Z, Kusumanchi P, Ross RA, Heathers L, Chandler K, Oshodi A, et al. Serum Metabolomic Profiling Identifies Key Metabolic Signatures Associated With Pathogenesis of Alcoholic Liver Disease in Humans. Hepatol Commun. 2019;3(4):542-57.

32. Marderstein EL, Bucher B, Guo Z, Feng X, Reid K, Geller DA. Protection of rat hepatocytes from apoptosis by inhibition of c-Jun N-terminal kinase. Surgery. 2003;134(2):280-4.

33. Liu P, Li R, Antonov AA, Wang L, Li W, Hua Y, et al. Discovery of Metabolite Biomarkers for Acute Ischemic Stroke Progression. J Proteome Res. 2017;16(2):773-9.

34. Sun $\mathrm{H}$, Zhao J, Zhong D, Li G. Potential serum biomarkers and metabonomic profiling of serum in ischemic stroke patients using UPLC/Q-TOF MS/MS. PLoS One. 2017;12(12):e0189009.

35. Alewijnse AE, Peters SL. Sphingolipid signalling in the cardiovascular system: good, bad or both? Eur J Pharmacol. 2008;585(2-3):292-302.

36. Niebergall LJ, Jacobs RL, Chaba T, Vance DE. Phosphatidylcholine protects against steatosis in mice but not non-alcoholic steatohepatitis. Biochim Biophys Acta. 2011;1811(12):1177-85. 
37. Chung SY, Moriyama T, Uezu E, Uezu K, Hirata R, Yohena N, et al. Administration of phosphatidylcholine increases brain acetylcholine concentration and improves memory in mice with dementia. J Nutr. 1995;125(6):1484-9.

38. Ramstedt B, Slotte JP. Membrane properties of sphingomyelins. FEBS Lett. 2002;531(1):33-7.

39. Vance JE, Tasseva G. Formation and function of phosphatidylserine and phosphatidylethanolamine in mammalian cells. Biochim Biophys Acta. 2013;1831(3):543-54.

40. Lind L, Salihovic S, Ganna A, Sundstrom J, Broeckling CD, Magnusson PK, et al. A Multi-Cohort Metabolomics Analysis Discloses Sphingomyelin (32:1) Levels to be Inversely Related to Incident Ischemic Stroke. J Stroke Cerebrovasc Dis. 2020;29(2):104476.

\section{Figures}

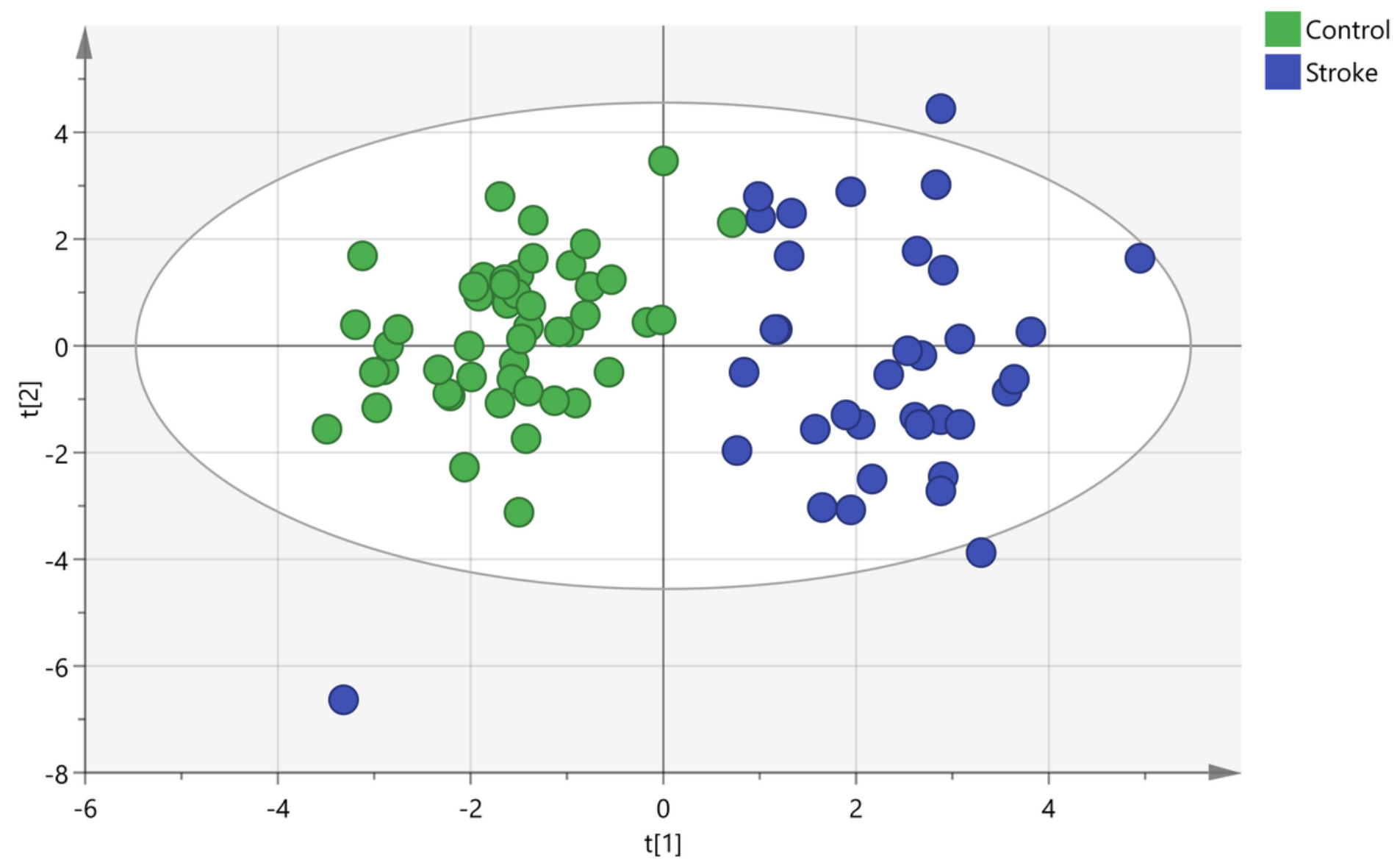

\section{Figure 1}

PCA score plot of the control and stroke groups. The cumulative fitness (R2 value) of the PCA model was 0.767. 

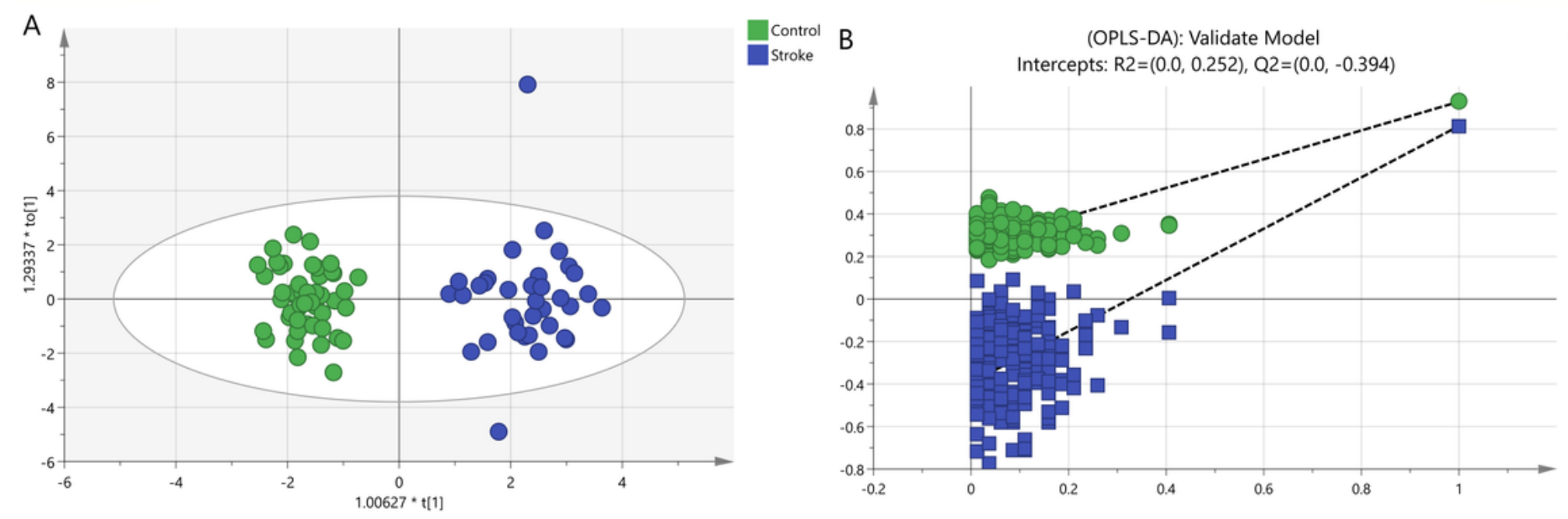

\section{Figure 2}

OPLS-DA score plot of the control and stroke groups. (A) OPLS-DA score plot of the control (green dots) and stroke (blue dots) groups ( $R 2 Y=0.928, Q 2=0.814)$. The $t[1]$ and $t$ [2] values in the figures represent the scores of each sample for principal components 1 and 2, respectively. Each dot, square, or diamond on the plot represents a sample in the corresponding group. (B) Permutation plots for the OPLS-DA model showing R2 (green) and Q2 (blue) values. The results of the permutation test strongly indicate that the original model was valid (R2 intercept $=0.252$, Q2 intercept $=-0.394$ ). 

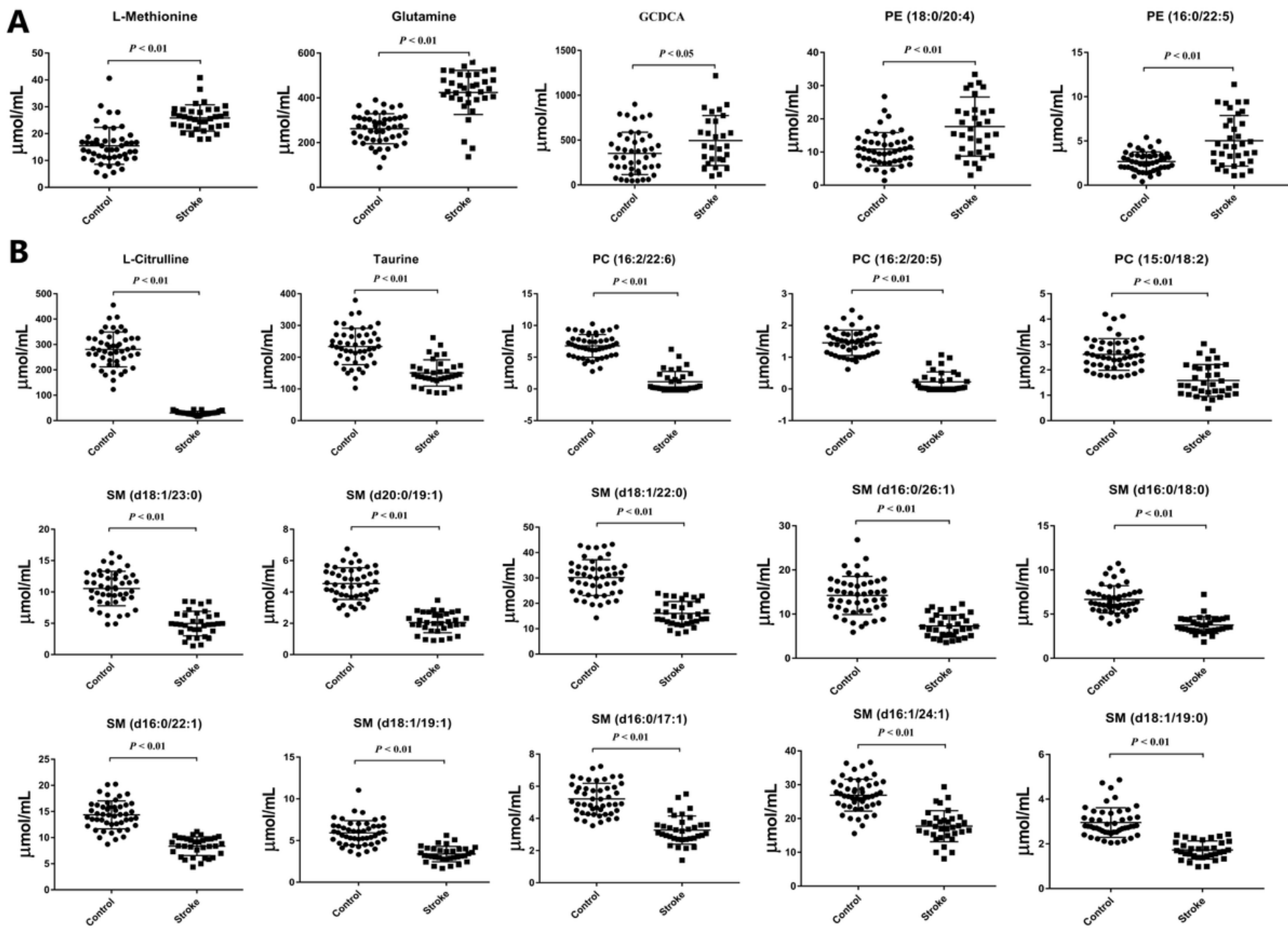

Figure 3

The significant changed metabolites between the control and stroke groups. (A) The stroke group had higher levels of L-methionine, glutamine, GCDCA, and PE (18:0/20:4, 16:0/22:5) compared to the control group. (B) The stroke group had decreased levels of L-citrulline, taurine, PC (16:2/22:6, 16:2/20:5, 15:0/18:2), and SM (d18:1/23:0, d20:0/19:1, d18:1/22:0, d16:0/26:1, d16:0/18:0, d16:0/22:1, d18:1/19:1, d16:0/17:1, d16:1/24:1, d18:1/19:0) compared to the control group. GCDCA: glycochenodeoxycholic acid; PE: phosphatidylethanolamine; PC: phosphatidylcholine; SM: sphingomyelin. 


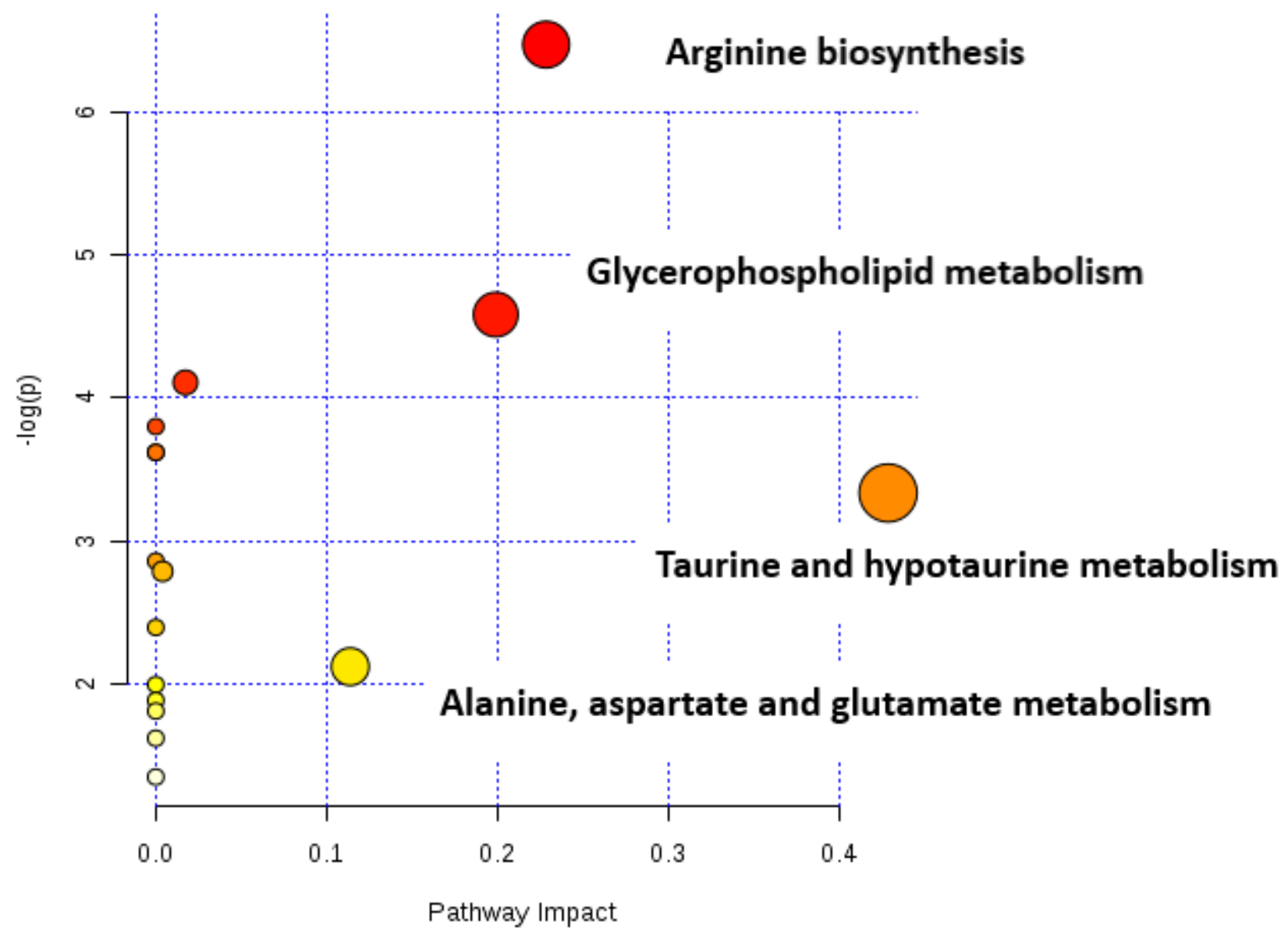

Figure 4

Pathway analysis of serum metabolite profiles of the stroke group compared to the control group. Pathway impact values are plotted against $X$-axis, and - $\log (P)$ values are plotted against $Y$-axis. For visual clarification, the pathway importance and the statistical significance are proportional to node radius and color, respectively.

\section{Supplementary Files}

This is a list of supplementary files associated with this preprint. Click to download.

- Supplementarydata.docx 